\title{
A Simplified Suggested Methodology for Quality Improvement in Radiation Oncology Facility
}

\author{
(1) Ahmed Nadeem ABBASI,' (1) Muneeb uddin KARIM,' (D) Bilal Mazhar QURESHI' \\ 'Department of Oncology, The Aga Khan University, Radiation Oncology Section, Karachi-Pakistan
}

\begin{abstract}
SUMMARY
Radiation oncology is a very quality conscious area of healthcare. Radiotherapy-related errors are not uncommon; moreover, many errors are likely to go undetected. Radiotherapy error can lead to the wrong, ineffective, or lack of delivery of a required radiation dose to the the target tissue. Quality assurance in radiation therapy includes procedures that ensure a consistent and safe fulfillment of dose prescription to the target volume, with a minimal dose to normal tissues and a minimal exposure to the personnel handling the equipment. In radiation therapy, a comprehensive quality assurance program is necessary because of the importance of accuracy in dose delivery. It reduces the likelihood of accidents and errors by increasing the probability of early detection of the errors that occur. Quality assurance in radiotherapy is concerned with all aspects of radiotherapy process and should involve all groups of staff in a cooperative approach, since quality activities are interdependent. The key component is a multidisciplinary approach that involves a radiation oncologist, a medical physicist, and radiation therapy technologists. This team ensures quality assurance that involves $3 \mathrm{Ms}$, i.e., machines, methodology, and manpower.
\end{abstract}

Keywords: Multidisciplinary approach; radiation therapy; quality assurance.

Copyright $\odot$ 2018, Turkish Society for Radiation Oncology

\section{Introduction}

A radiation oncology facility aims to provide the best possible quality service to the patients. In this era of advancing knowledge and precision technology, radiation treatment planning and delivery is required to have valid, tested, and proven quality assurance parameters encompassing all areas of a radiation therapy facility. Radiation therapy is considered as one of the quality conscious areas of healthcare. Right from multidisciplinary team decisions providing referral to radiation oncology, educating patient about radiotherapy, providing simulation, and achieving an appropriate, safe, and accurate treatment plan for the patient to a follow up plan and radiation protection for radiation personnel, well established international standards need to be followed in a robust manner. Monitoring the equipment for precise dose delivery and having adequate dose verification tools are equally important for a quality radiation oncology program.[1] Maintaining such consistency in all the areas of radiation oncology is very important to be part of a tertiary healthcare facility.

\section{Quality Assurance in Radiation Therapy:}

A comprehensive quality assurance system is required to guarantee the patients that these medical procedures will yield valuable information or provide needed treatment that can be appropriately implemented and used to achieve a positive impact on their health.[2] Radiation oncology is a very quality conscious department. All quality issues and systems can be appropri- 
ately managed if we think in terms of $3 \mathrm{Ms}$ of quality healthcare:
1. machines
2. methodology
3. manpower

In a modern radiation oncology facility, a quality improvement committee (QIC) is represented by all sections of the department. Sources of error in radiation therapy include tumor localization, lack or inappropriate patient immobilization, field placement and human errors in calibration, calculation in daily patient setup, and equipment-related problems. Many of these equipment and calculation errors can be minimized through a quality program comprising periodic checks.

A comprehensive quality assurance program in radiation therapy has both clinical and physical components. The production of radiation and the mechanical motions is controlled by numerous electrical control circuits. Quality assurance checks are necessary to verify the functionality of various backup systems to the primary control system. One of the most important backup systems available on all the therapy machines is the emergency system.

To ensure quality in healthcare sector, a radiation therapy unit should adhere to the following checklist:

- morning conference

- chart rounds

- plan checks

- treatment-machine check

- patient dose measurements

- beam profiles

These are some of the procedures that are recommended to assure that the radiation treatments are appropriately prescribed and accurately delivered.

- Morning conference: It is advisable to develop a peer review culture in the department. Every morning, physicians should convene for a peer review of the treatment plans. It will be desirable to meet in the form of a team and discuss patients seen and planned during the previous days. Physicians present the cases and the colleagues conduct a peer review of the cases.

- Chart rounds: The staff must meet every week to review the portal films and clinical condition of patients under treatment.

- Plan checks: A physicist must check the accuracy of the calculations and the details of the setup instructions.
- Chart checks: A physicist along with other physicists and radiation therapy technologists must weekly review the chart of every patient under treatment for continuous argument with the treatment plan.[3]

- Treatment-machine checks: Each morning, the radiation output from the linear accelerators must be measured and checked for consistency. The physicist must use additional equipment at weekly, monthly, and annual intervals to maintain the calibrations.

- Patient dose measurement: The radiation dose applied to selected patients must be measured to verify the calculations.

- Portal films: Before the treatment begins and at least weekly thereafter, films are obtained using treatment beams to verify the continual accuracy of the dose delivered. Radiation treatment delivery software must check the output of each beam of radiation treatment delivery, and therapists must check all settings against the original plan.[4,5]

\section{Measuring the Quality:}

An important aspect of quality assurance is a clinical quality indicator (CQI). A quality indicator is a measure of an important aspect of the quality of care or the quality of services provided. It is not a direct measure of quality but acts as a screening tool or reference point for monitoring, maintaining, and evaluating and improving care. Both CQIs and non-CQIs are used and include those that relate to the structure function, process, and outcome. In general, indicators should represent those procedures, conditions, or services that are

- frequently occurring;

- easy to monitor through the collection of readily accessible data;

- high risk, high volume, high cost, or problem prone;

- amenable to intervention; and

- highly variable in the way they are managed.[6]

Clinical and physical quality indicator data should be aggregated over time to show trends and patterns. The selection of indicators is an agenda item of the QIC. It is important to remember that indicators do not provide definitive answers about the quality rather prompt us to question the occurrence of particular events.

Quality assurance and quality control are essential components of the work performed by the medical 
physics team. This team provides support to the department of radiation oncology by providing

- quality control of radiation therapy treatment units,

- technical support and quality control for brachytherapy, and

- Thermoluminescent dosimeter (TLD) monitoring for clinical and research activities.

\section{Management of Quality Assurance:}

The role of the QIC in managing all aspects of departmental quality assurance is crucial. Irrespective of the name, this board bears the ultimate responsibility for the quality of patient care rendered within the facility. The key responsibilities of QIC in quality area include the follow

- maintaining a coordinated system while approaching toward improving patient care and health outcomes,

- understanding the board's role in the performance of the improvement program,

- approval of written performance improvement or quality assessment plan,

- regularly reviewing the results of performance improving activities,

- explaining the hospitals' performance improvement program and how quality in patient care is achieved, and

- familiarity with external audit systems, such as JCIA and ISO.

\section{Conclusion}

The treatment procedure of radiotherapy is a complex and critical process. This process involves the understanding of different integrated steps that include the principles underlying medical physics, radiobiology, radiation safety, radiation treatment planning, and radiation interaction with other treatment modalities. To manage all the complexities related to new advanced technologies, radiation oncologists have to enhance their professional skill profiles. Each step in the integrated process of radiotherapy needs quality control and quality assurance to prevent errors and to provide high assurance that patients will accurately receive the prescribed treatment.[7]
The key component is a multidisciplinary approach, which involves a radiation oncologist, a medical physicist, and radiation therapy technologists. This team ensures the quality assurance that involves the $3 \mathrm{Ms}$.

Peer-review: Externally peer-reviewed.

Conflict of Interest: None declared.

Authorship contributions: Concept - A.N.A.; Supervision - A.N.A.; Data collection \&/or processing - Not applicable; Literature search - A.N.A., M.u., B.M.Q.; Critical review A.N.A., M.u., B.M.Q.

\section{References}

1. Abbasi AN. Establishment and Maintenance of Quality of Site-Specific Multidisciplinary Tumor Boards in Pakistan. J Coll Physicians Surg Pak 2016;26(10):805-7.

2. Valentini V, Glimelius B, Frascino V. Quality assurance and quality control for radiotherapy/medical oncology in Europe: guideline development and implementation. Eur J Surg Oncol 2013;39(9):938-44.

3. Pham N, Asper J, Bonnen M, Mok H, Wagner T, Ludwig $M$, et al. Pre-treatment peer-review: enhancing value through increased efficiency and effectiveness of radiation oncology peer review. Journal of Radiation Oncology 2018;7(1):97-102.

4. Lefresne S, Olivotto IA, Joe H, Blood PA, Olson RA. Impact of quality assurance rounds in a Canadian radiation therapy department. Int J Radiat Oncol Biol Phys 2013;85(3):e117-21.

5. Nadeem Abbasi A, Muhammad W, Hussain A. Implementation of quality medical physics training in a lowmiddle income country - sharing experience from a tertiary care JCIA-accredited university hospital. J Appl Clin Med Phys 2016;17(6):454-6.

6. Rosenblatt E, Fidarova E, Ghosh S, Zubizarreta E, Unterkirhere O, Semikoz N, et al. Quality of radiotherapy services in post-Soviet countries: An IAEA survey. Radiother Oncol 2018;127(2):171-7.

7. Harris J, Green JS, Sevdalis N, Taylor C. Using peer observers to assess the quality of cancer multidisciplinary team meetings: a qualitative proof of concept study. J Multidiscip Healthc 2014;7:355-63. 